\title{
The Potential of Rotating-Baseline Nulling Interferometers Operating within Large Single-Telescope Apertures
}

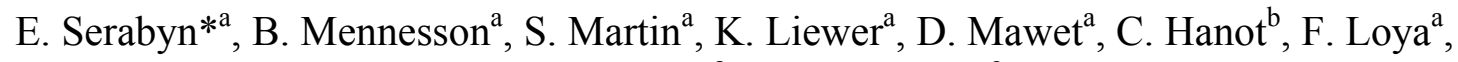 \\ M.M. Colavita ${ }^{\mathrm{a}}$ and S. Ragland ${ }^{\mathrm{c}}$ \\ a Jet Propulsion Laboratory, 4800 Oak Grove Drive, \\ California Institute of Technology, Pasadena, CA, USA 91109; \\ ${ }^{\mathrm{b}}$ University of Liege, Allee du 6 Aout, 17 Bat B5c, 4000 Liege, Belgium \\ ${ }^{\mathrm{c}}$ W. M. Keck Observatory, Kamuela, HI 96743
}

\begin{abstract}
The use of a rotating-baseline nulling interferometer for exoplanet detection was proposed several decades ago, but the technique has not yet been fully demonstrated in practice. Here we consider the faint companion and exozodiacal disk detection capabilities of rotating-baseline nulling interferometers, such as are envisioned for space-based infrared nullers, but operating instead within the aperture of large single telescopes. In particular, a nulling interferometer on a large aperture corrected by a next-generation extreme adaptive optics system can provide deep interferometric contrasts, and also reach smaller angles (sub $\lambda / \mathrm{D}$ ) than classical coronagraphs. Such rotating nullers also provide validation for an eventual space-based rotating-baseline nulling interferometer. As practical examples, we describe ongoing experiments with rotating nullers at Palomar and Keck, and consider briefly the case of the Thirty Meter Telescope.
\end{abstract}

Keywords: nulling interferometry

\section{INTRODUCTION}

Great progress is being made in the detection and characterization of planets orbiting nearby stars with radial velocity and transit measurements, but these techniques are sensitive mainly to very close-in planets. On the other hand, coronagraphy, which aims at the high-contrast imaging of solar systems, is most sensitive at larger distances from stars (typically beyond angles of a few $\lambda / \mathrm{D}$ of a star, where $\lambda$ is the wavelength, and $\mathrm{D}$ the telescope diameter). Interferometry can bridge the gap between these approaches, providing higher angular resolution than direct imaging, and reaching smaller angles than coronagraphy. However, contrasts achieved with long-baseline interferometry have to date been fairly modest. On the other hand, planned next-generation "extreme" adaptive optics (ExAO) systems should enable improved interferometric contrasts across large single-telescope apertures. Moreover, the possibility of ExAO-corrected 30-40 m class telescopes in the next decade suggests a very significant potential for "cross-aperture" interferometry.

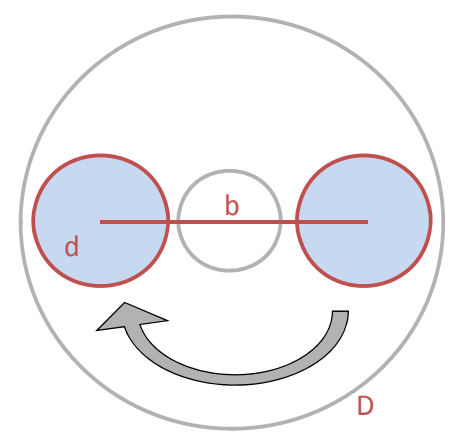

Figure 1. Schematic of an interferometer with a rotating baseline of length $b$ and subaperture diameter $d$ within a single telescope aperture of diameter D.

*gene.serabyn@jpl.nasa.gov; phone 1818 393-5243; fax 1818 393-5243

Optical and Infrared Interferometry II, edited by William C. Danchi, Françoise Delplancke, Jayadev K. Rajagopal, Proc. of SPIE Vol. 7734, 77341E · (c) 2010 SPIE · CCC code: 0277-786X/10/\$18 · doi: 10.1117/12.857753 
High-contrast interferometric observations of faint sources near bright stars require either exquisitely well-calibrated visibilities or a reduction of the stellar flux prior to detection, by means of interferometric nulling. The use of a spacebased separated-aperture rotating-baseline nulling interferometer for the detection of exoplanets was originally proposed by Bracewell ${ }^{1}$, but this approach has not yet been demonstrated fully on the sky. However, the efficacy and potential of this approach can be demonstrated quite simply, by rotating a nulling baseline within the aperture of a large telescope (Fig. 1). For the past several years we have therefore been pursuing the development of such rotating nullers at the Palomar and Keck Observatories ${ }^{2-5}$. In this paper, we discuss the novel observational parameter space opened up with such rotating nullers, the development steps and experiments that we have undertaken to date, and the unique astronomical observations of faint companions and extended circumstellar emission that such systems can enable.

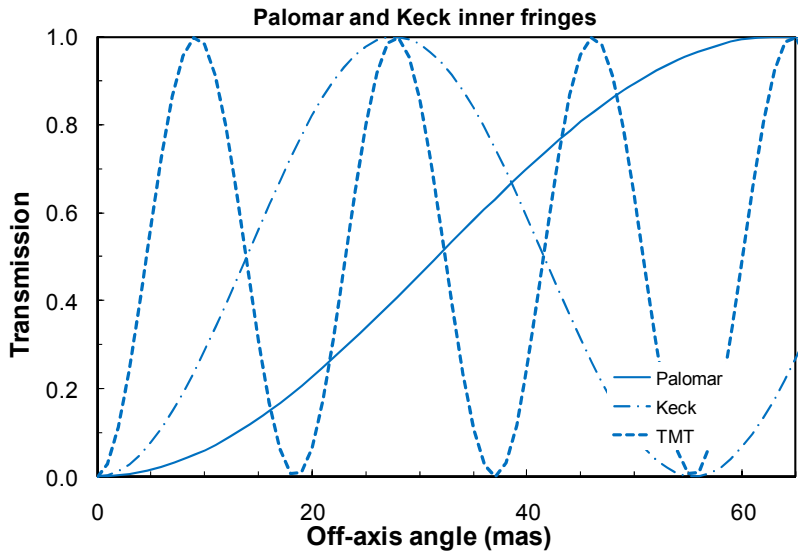

Figure 2. Transmissions of the innermost K-band fringes for a dual-aperture nuller at the Palomar, Keck and TMT telescopes, for baselines of 3.4, 8.5 and $28.5 \mathrm{~m}$, respectively. To half power transmission, a single Keck telescope equipped with a rotating K-band nuller can observe to within $\sim 13$ mas of stars $(0.13 \mathrm{AU}$ for a star at $10 \mathrm{pc})$.

\section{BASIC INTERFEROMETRY PARAMETERS}

To reduce the stellar flux across a broad band, a nuller must generate a deep achromatic "null" interference fringe across the center of the star. A rotating nuller must furthermore establish a centered null over the baseline rotation angles sampled, but in practice the baseline can be stepped in azimuth and the nuller rephrased at each step. With an achromatic phase shift of $\pi$ radians between a pair of combining beams, the monochromatic dual-aperture intensity fringe pattern is

$$
\mathrm{I}=\mathrm{I}_{0} \sin ^{2}(\pi \mathrm{b} \theta / \lambda)
$$

for which the angular resolution, $\theta_{\mathrm{R}}$, is $\lambda / 2 \mathrm{~b}$. The maximum baseline available across a single aperture of diameter $\mathrm{D}$ is $D-d$, where $d$ is the individual subaperture diameter, so that the best angular resolution is $\lambda / 2(D-d)$, which approaches $\lambda / 2 \mathrm{D}$ (a factor of 2 better than with full aperture imaging) as the subaperture diameter goes to zero. The innermost half power transmission point of the first fringe occurs for

$$
\theta_{\mathrm{H}}=\lambda / 4 \mathrm{~b},
$$

so that the half power angle (the "inner working angle," or IWA) for the longest baseline is given by

$$
\theta_{\mathrm{H}}=\lambda / 4(\mathrm{D}-\mathrm{d})
$$

For illustrative purposes, if we take $\mathrm{d}=\mathrm{D} / 4$, we get

$$
\theta_{\mathrm{H}}=\lambda / 3 \mathrm{D},
$$


which is significantly smaller than the few $\lambda / \mathrm{D}$ inner working angles of classical single-aperture coronagraphs ${ }^{6}$. The halfpower points for the cases of $1.5 \mathrm{~m}$ subapertures on the Palomar $5 \mathrm{~m}$, Keck $10 \mathrm{~m}$, and planned Thirty Meter Telescope (TMT) in $\mathrm{K}_{\mathrm{s}}$ band $(2.15 \mu \mathrm{m})$ are then $\sim 33$ mas, 13 mas, and 3.9 mas respectively (Fig. 2$)$. These very small IWAs allow observations of nearby solar systems on sub-AU scales out to several tens to hundreds of parsecs, resp.

Of course, the light from off-center sources will pass through the regions of constructive interference fringes. Off-axis emission sources are then detectable because as the off-axis fringes rotate about the center, they sweep past and modulate such emission (Fig. 3). The signal shapes and power spectra vary as a function of radial distance, while the timing reflects the source azimuthal structure, enabling source structure to be reconstructed in detail ${ }^{7}$ (Fig. 3).
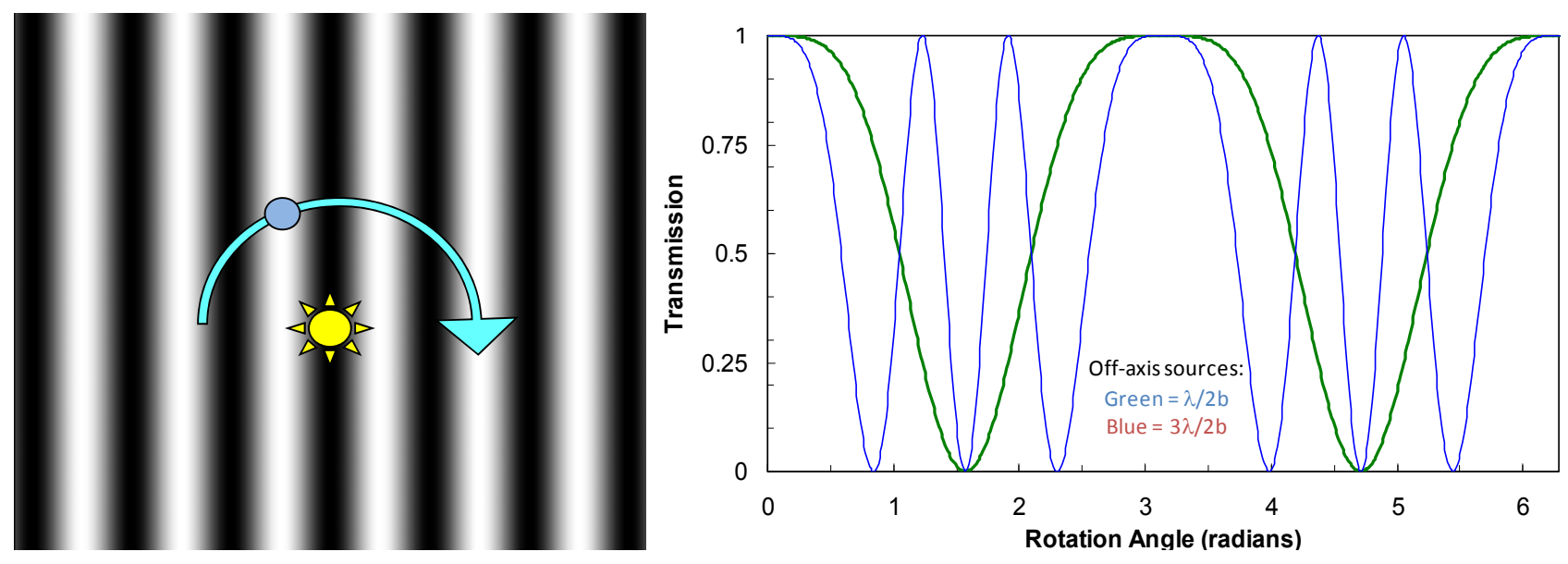

Figure 3. Left: Schematic of the measurement approach. The star is kept centered on the null fringe, and as the baseline rotates, fringes sweep past and modulate off-axis sources. Right: Transmission vs. time for companions at two different distances $(\lambda / 2 b$ and $3 \lambda / 2 b)$.

The other angular scale to consider is the outer working angle, $\theta_{\mathrm{O}}$, which is defined as half the subaperture's singlemode field of view (FOV), i.e.,

so that

$$
\theta_{\mathrm{O}}=\theta_{\mathrm{FOV}} / 2=\lambda / 2 \mathrm{~d}
$$

$$
\theta_{\mathrm{O}} / \theta_{\mathrm{H}}=\theta_{\mathrm{FOV}} / \theta_{\mathrm{R}}=2 \mathrm{~b} / \mathrm{d},
$$

implying that there are only a few resolution elements across the field of view unless $\mathrm{d}<<\mathrm{D}$. For the longest possible baseline, this becomes

$$
\theta_{\mathrm{O}} / \theta_{\mathrm{H}}=2(\mathrm{D} / \mathrm{d}-1)
$$

In our $\mathrm{D} / \mathrm{d}=4$ example, there are then 6 resolution elements across the FOV, while in the real case of the Palomar Fiber Nuller $(\mathrm{PFN})$, where $\mathrm{d}$ was chosen to be as large as possible $(1.5 \mathrm{~m})$ so as to maximize the signal, $\theta_{\mathrm{O}} / \theta_{\mathrm{H}}=4$. On the other hand, for the same subaperture size in the TMT case, $\theta_{\mathrm{O}} / \theta_{\mathrm{H}}$ could be as large as $\sim 40$, thus potentially providing high-quality, high resolution images. Note that the outer working angle can be rewritten

$$
\theta_{\mathrm{O}}=(\mathrm{D} / 2 \mathrm{~d}) \lambda / \mathrm{D}
$$

so that for small $\mathrm{D} / \mathrm{d}$ (of order a few), the interferometric outer working angle is comparable to typical coronagraphic inner working angles ${ }^{6}$, thus enabling such nullers to uniquely access very small angles. 


\section{NULLING BEHIND AN ADAPTIVE OPTICS SYSTEM}

Deep starlight nulling requires a high degree of symmetry and stability ${ }^{8}$. Combining the light from a pair of subapertures within a large telescope aperture has the potential to provide the needed symmetry, because most of the optical path is common mode, while stability, in both relative phase and relative intensity, can be provided by an adaptive optics system. Indeed, residual phase errors behind next-generation ExAO systems $(<100 \mathrm{~nm})$ are expected to be significantly lower than those typical of long-baseline separated-aperture interferometers. Thus, even relatively short (i.e., near-IR) wavelengths can be nulled to significant levels by making use of an ExAO system as a fringe tracker (and including a relative $\pi$ phase shift). Given this potential, we have been developing rotating-baseline nulling interferometry techniques in the near-IR, in order to demonstrate companion detection with this technique, and to provide unique access to regions very near stars.

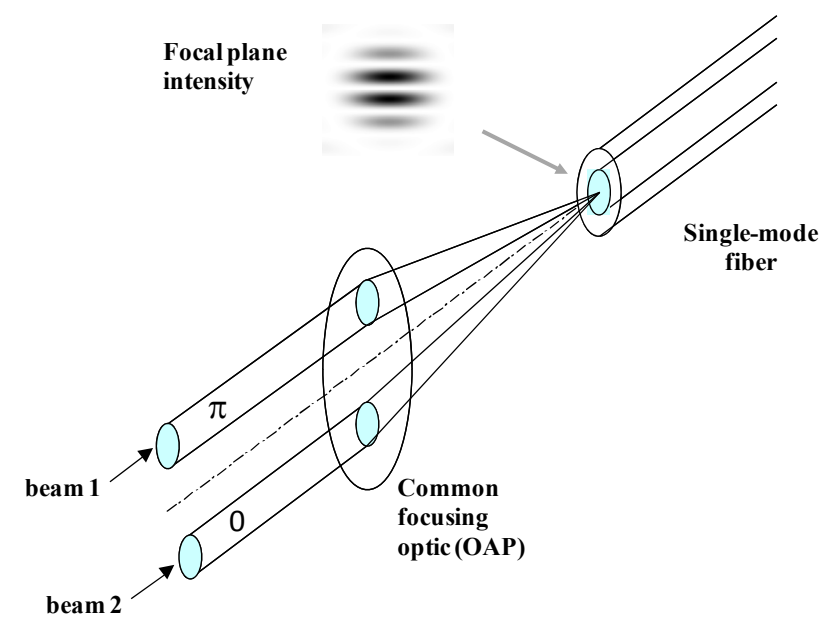

Figure 4. Conceptual layout and operation of a fiber nuller: because of destructive interference, the on-axis starlight cannot propagate in the single-mode fiber ${ }^{9}$. The Palomar Fiber Nuller (PFN) uses an off-axis paraboloid $(\mathrm{OAP})$ as the focusing element, and the combination of a split-mirror and tiltable dielectric plates to introduce an achromatic $\pi$-radian phase shift.

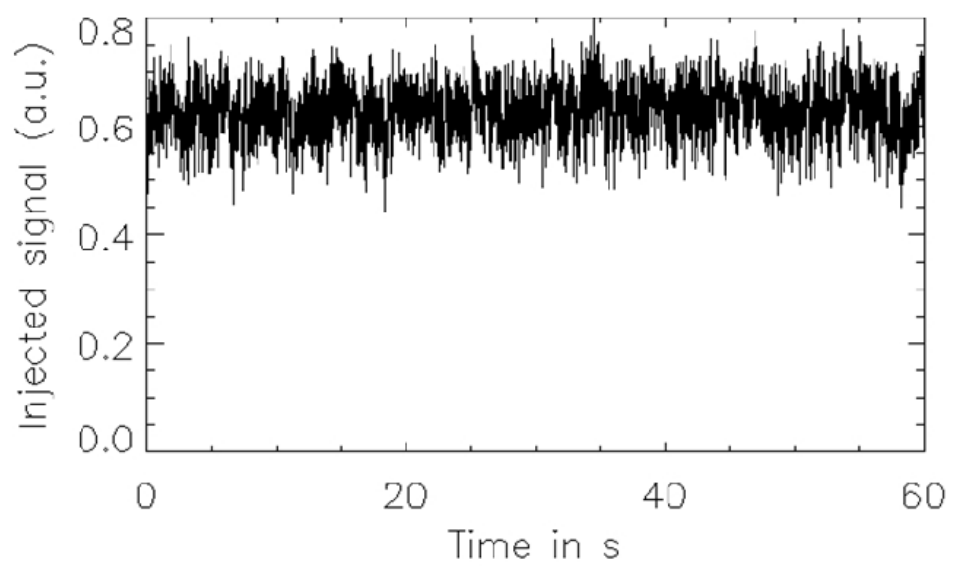

Figure 5. Measured coupling on the sky at Palomar of one $3 \mathrm{~m} \mathrm{x} 1.5 \mathrm{~m}$ beam into the PFN fiber as a function of time (while angle-tracking). The observed fluctuations correspond directly to the temporal variations of the Strehl ratio after $\mathrm{AO}$ correction. They are measured here to be $7 \% \mathrm{rms}$ over $1 \mathrm{~min}$. Intensity mismatches between the two beams have limited impact on the observed null (second order effect). These are monitored on short time scales with our fast beam chopper and most of their effect is taken out by our statistical data reduction approach. 

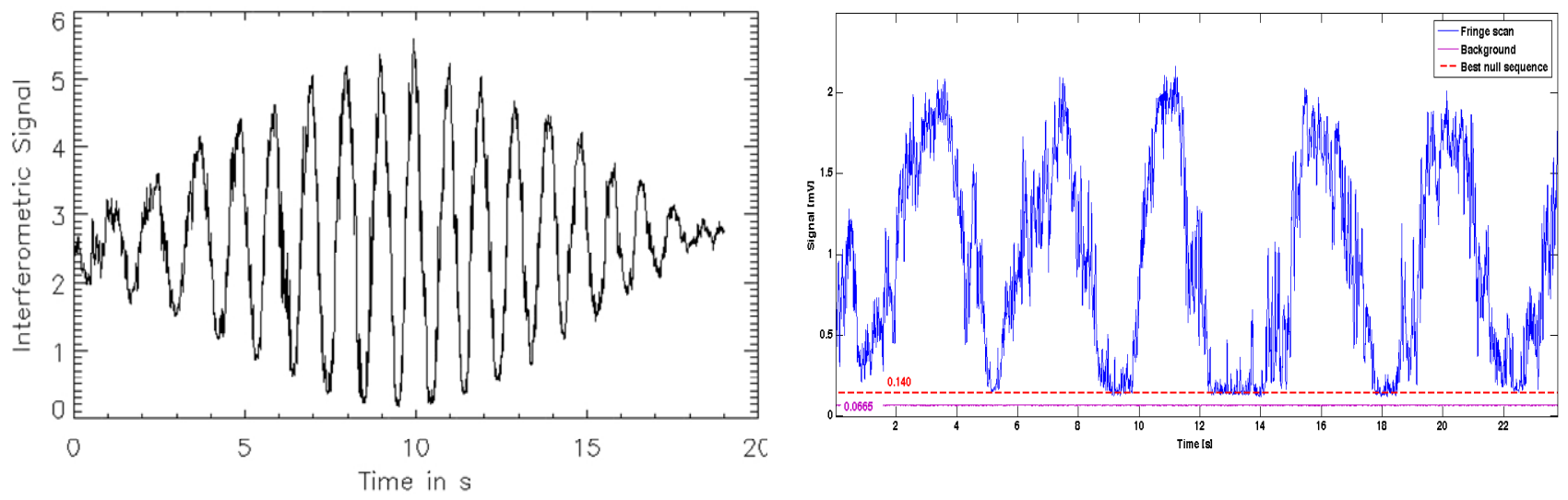

Figure 6. Left: Slow scan through the fringe packet obtained on $\alpha$ Her using only the current Palomar AO system as the upstream "fringe tracker". The chirp evident near the left-hand end of the scan likely arises in PZT nonlinearities. Right: Slow fringe scan through the central few fringes, showing the effect of the resolved star on the fringe minima: the central few fringes have well defined minima.

\subsection{Palomar}

At Palomar, we are demonstrating simplified nulling beam combination (Fig. 4) via a single-mode fiber ${ }^{9}$ (the Palomar Fiber Nuller ${ }^{2-5}$ or PFN), as well as a new statistical data analysis approach that allows the astronomical null depth to be determined accurately even in the presence of fluctuations ${ }^{10,11}$. The basic idea is that the square-law dependence of null depth on phase errors (valid near the fringe minimum) translates directly into a recognizable signature in the distribution of null depths observed in a time sequence. In particular, the expected null depth distribution becomes significantly asymmetrical, in a manner which can be calculated analytically in the simplest case of pure phase errors ${ }^{10}$.
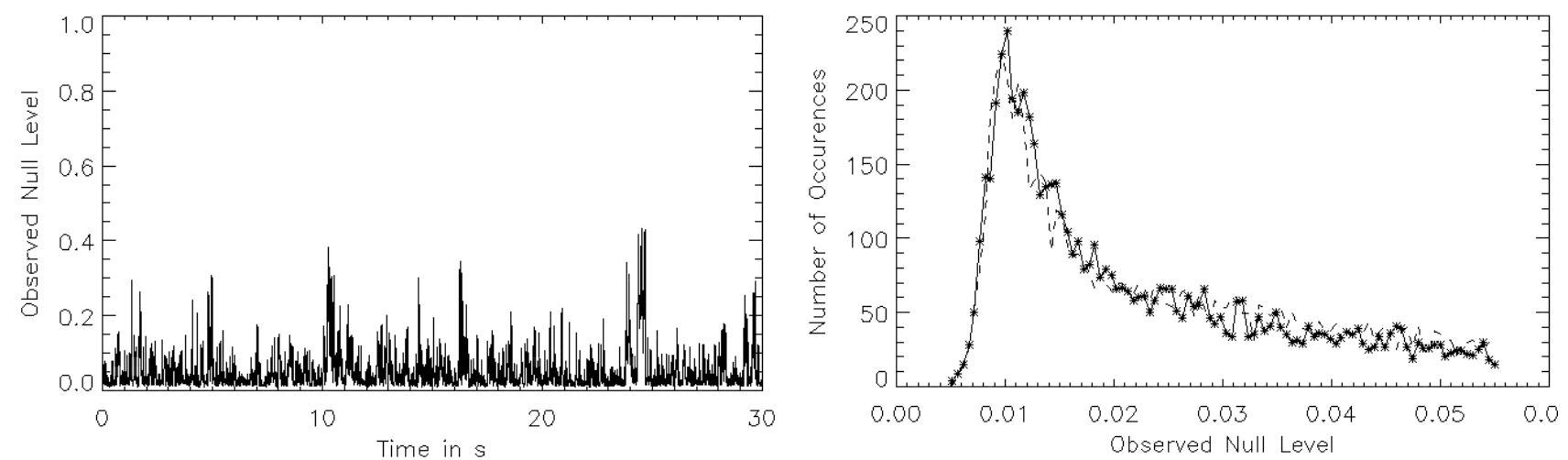

Figure 7. Left: A time sequence of null measurements obtained with the Palomar Fiber Nuller on $\beta$ Peg. Note the very one-sided noise distribution above the minimum null value. Right: Comparison of the distribution of $\beta$ Peg null depth measurements with our statistical model for the expected null depth distribution.

Our initial observations at Palomar have begun to demonstrate the efficacy of our new hardware and software techniques, as well as of the use of relatively short near-IR nulling baselines. In particular, our statistical data analysis approach allows measurement of much deeper nulls than the stabilization level suggests. Fig. 5 shows the single-beam intensity stability achieved at Palomar on a night of good seeing, while Fig. 6 (left) shows a slow scan through a fringe packet on $\alpha$ Her, showing remarkable fringe stability. Fig. 6 (right) shows the effect of a resolved star: the fringe minima are limited to the astrophysical null depth, which can essentially be read off the raw data plot without any calibration. 


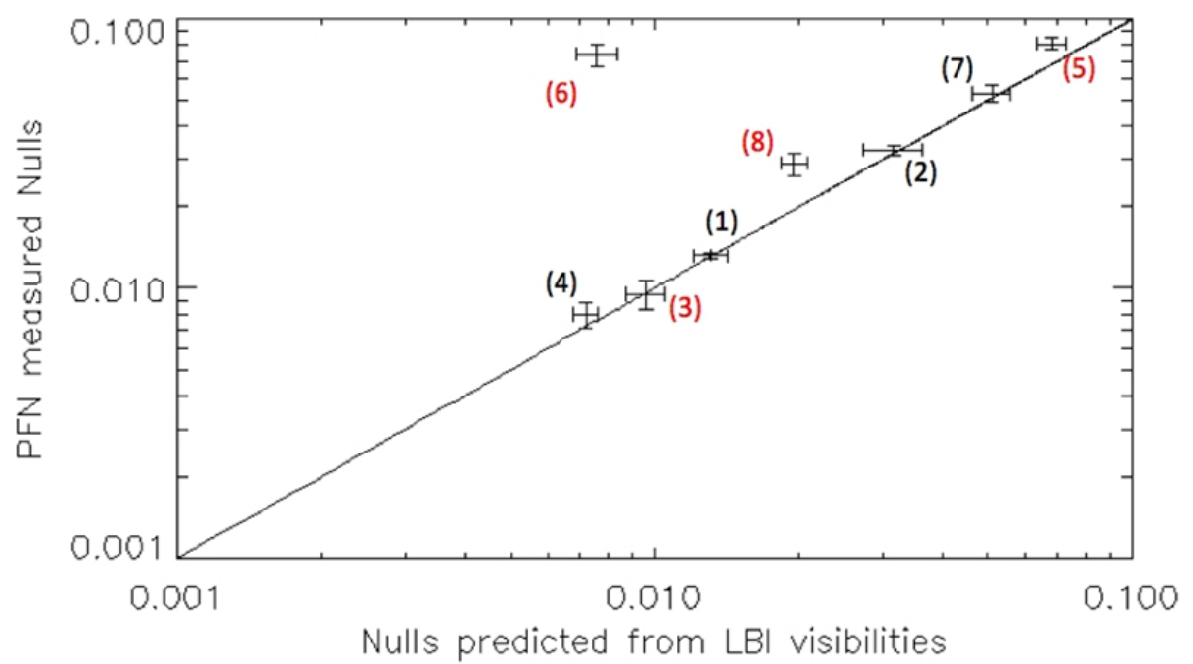

Figure 8. Null depths measured ${ }^{11}$ by the Palomar Fiber Nuller on several evolved stars vs. the values expected from near IR long baseline interferometry and stellar photosphere modeling (no extended shells included). (1): $\alpha$ Boo (Perrin et al. 1998, Lacour et al. 2008), (2): $\alpha$ Her (Perrin et al. 2004), (3): $\beta$ Peg (Di Benedetto \& Rabbia 1987), (4): $\beta$ And (Richichi et al. 2005), (5): $\alpha$ Ori (Perrin et al. 2004), (6): $\rho$ Per (Richichi et al. 2005), (7): $\alpha$ Aur (Torres et al. 2009, Hartkopf \& Mason 2006), (8): $\chi$ Cyg (Lacour et al. 2009). The agreement is generally excellent for standard $\mathrm{K}$ giants and supergiants (black). Departure from naked photosphere models is only found for three of the Miras and semi-regular variable stars (red), which exhibit outer molecular layers and dust shells ( $\alpha$ Ori, $\rho$ Per and $\chi$ Cyg). The large discrepancy measured on $\rho$ Per suggests a recent mass loss event.

However, even so, extreme levels of phase matching would be required to achieve extremely stable, deep nulls. On the other hand, even without a highly stabilized null, one can just sit near null, and record the null depth variations as fluctuations move the system back and forth across null (Fig. 7, left). As can be seen in Fig. 7 (right), the distribution of these measured nulls can be fitted very accurately with our statistical treatment ${ }^{10}$ of the fluctuating null depth, allowing high null depth accuracy to be attained, and intrinsically deep astrophysical nulls to be recovered. Our initial Palomar results include the measurement of the diameters of several evolved stars to high accuracy even with our short $3.4 \mathrm{~m}$ baseline $^{11}$ (Fig. 8), and the measurement of a null depth on $\mathrm{Vega}^{12}$ at approximately the $0.1 \%$ level (Fig. 9).

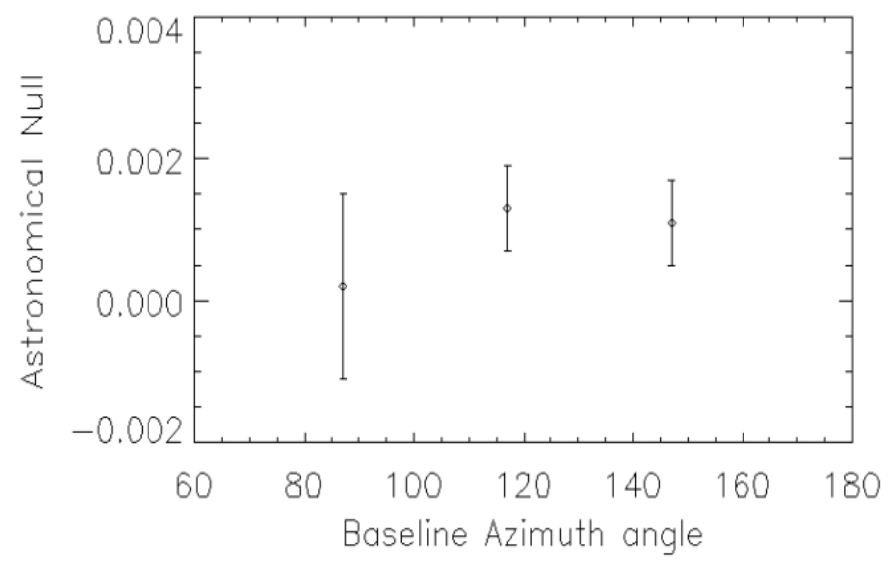

Figure 9. Null depths measured vs. baseline azimuth on Vega at Palomar ${ }^{12}$. The rms null measurement accuracy is $\sim 0.06 \%$. 


\subsection{Keck}

However, as our Palomar baseline ${ }^{11}$ is only $3.4 \mathrm{~m}$, we have also carried out initial engineering tests at Keck, in order to evaluate its potential in this regard. At Keck, to a large extent we make use of existing Keck Interferometer infrastructure, but combine beams across an aperture instead of between apertures (and use only one telescope). These tests have verified that cross-aperture fringes are indeed already very stable (Fig. 10), $120 \mathrm{~nm}$ rms, thus potentially allowing for stabilized nulls almost to the $10^{-2}$ level. At Keck, the fringes are stabilized first by the AO system, and then improved by the existing basement fringe tracker. Note that there is currently no K-band nuller available at Keck, so our current Keck measurements rely on normal visibility measurements.

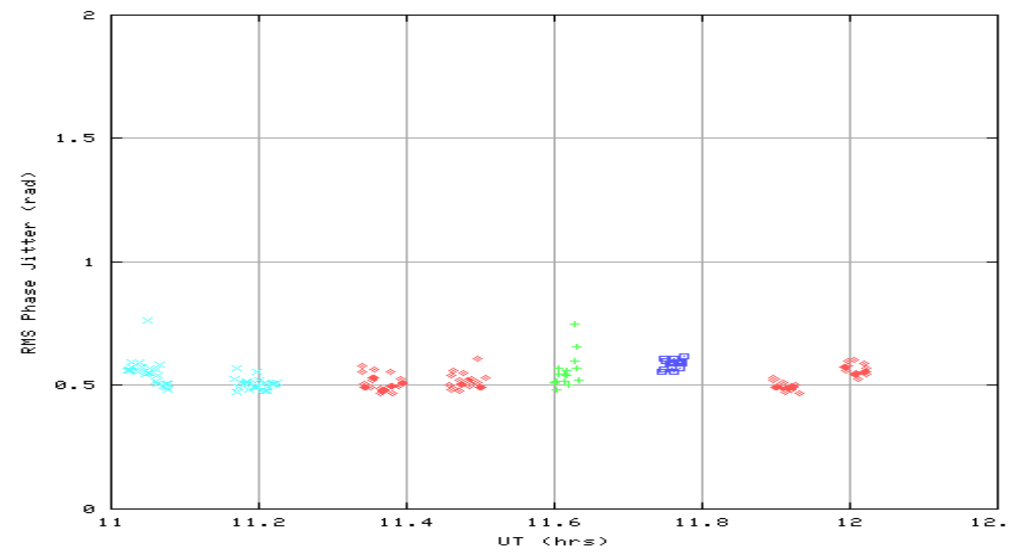

Figure 10. Cross-aperture rms phase jitter obtained at Keck. Together with spectral analysis of the residual jitter, roughly $120 \mathrm{~nm}$ can be expected, limited mostly by internal vibrations.

Even with these limitations, test observations of a binary star at Keck, obtained by rotating the baseline with the Keck AO system's K-mirror, have already resulted in the demonstration of companion detection at modest contrasts (Fig. 11). Thus, with a true near-IR nulling beamcombiner, some modifications to the control software, and the use of our statistical data analysis techniques for retrieving null depths in the presence of fluctuations, significantly fainter companion detections should be possible.

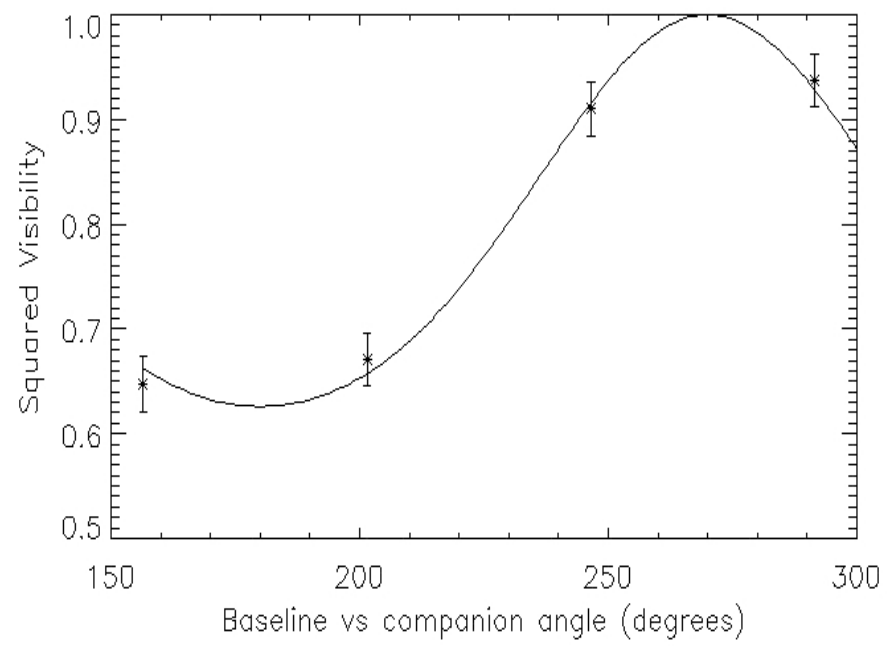

Figure 11. Detection of a faint binary companion (HIP 87895, with $\Delta \mathrm{K}=2.1$ mag. and separation $=39$ mas) with a single aperture rotating baseline interferometer at Keck. In contrast, the calibrator star gave a very flat response vs. angle. 

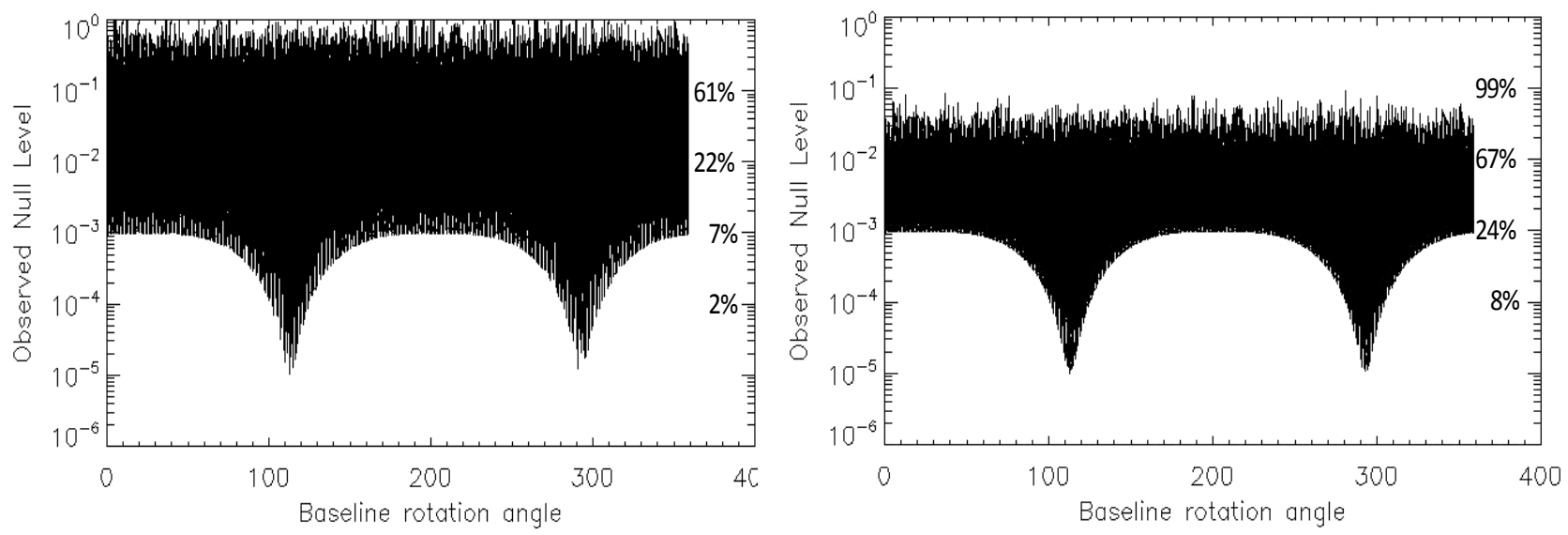

Figure 12. Predicted null signal modulation at Keck (compare Fig. 3) expected for one nuller baseline rotation (with 1000 individual reads) for a binary with a $\mathrm{K}$ band flux ratio of $10^{-3}$ and $60 \mathrm{mas}$ separation. At a given baseline orientation, the measured null depth fluctuates due to residual phase jitter between the two beams. The percentage time spent below the $10^{-\mathrm{n}}$ null level is shown on the right hand vertical scales. Left: Present-generation AO with $\sim 200 \mathrm{~nm}$ rms jitter. Right: ExAO with 50nm rms jitter. For each baseline angle, the deepest null measurable depends on the orientation of the baseline with respect to the binary separation vector. As the companion crosses regions of low and high transmissions in the rotating sky fringe pattern, the deepest measurable null value is correspondingly modulated. It varies between the instrumental limit (for a baseline perpendicular to the binary) and the binary contrast ratio (baseline parallel to the separation vector). Note that with ExAO (right), the mean null level and the top envelope of the fluctuations drop significantly, and much more time is spent in the vicinity of deep nulls, making companion detection faster and flux characterization more accurate.

Figure 12 shows our model predictions for the expected K-band null depth measurements on a binary star at Keck for a single rotation of a nulling baseline, for both current AO correction levels (left) and ExAO correction (right). Also given along the right hand edge of each plot is the time fraction for which the measured nulls are expected to be below the corresponding $10^{-\mathrm{n}}$ levels. Including our statistical analysis method, our modeling suggests that near-IR null depth measurements as low as $10^{-4}$ should be possible on bright stars at Keck. Such deep nulling, combined with the small inner working angle, will enable access to a unique region of observational parameter space, thus providing unique information on the presence of brown dwarfs and exozodiacal dust in the innermost regions of nearby solar systems. Young hot jupiters should be possible as well, but hot jupiters around evolved stars most likely require somewhat longer wavelengths (L band).

\section{THE POTENTIAL}

We are still in the initial phases of developing and modeling rotating nullers, but we can already predict that state of the art sensitivities on large telescopes should enable null depth measurements of order $10^{-4}$ on bright stars, thus allowing the imaging of the innermost regions of nearby solar systems, where both planets and dust are brighter than at larger radii. Especially promising of course are young planets. However, another interesting case is that of exozodiacal dust, as recent near-IR long-baseline interferometry results suggest $\sim 1 \%$ excesses in the immediate vicinity of several main sequence stars ${ }^{13-17}$ : the A-stars Vega, $\zeta$ Aquilae, $\beta$ Leo, Fomalhaut, as well as $\tau$ Ceti. These same stars thus far show no mid-IR excesses, thereby suggesting very hot $(1000-1500 \mathrm{~K})$ dust in the first AU around nearby main sequence stars. Such dust should be expelled by radiation pressure within only a few years, thus suggesting inordinate replenishment rates. The steady state collisional grinding of a massive asteroid belt thus cannot be the origin of such hot dust, which is more likely produced by isolated catastrophic events (e.g., a major asteroid collision or the break-up of a massive comet) or by major dynamical perturbations such as the Falling Evaporating Bodies phenomenon in the $\beta$ Pic inner disk, or the 
Late Heavy Bombardment that occurred early in the history of our solar system. However, the true nature of this close-in near-IR excess emission remains to be determined.

With good sampling of different orientations with baseline rotation, and different baseline lengths across the telescope aperture as well, a rotating nuller at Keck will be able to clarify the nature of the observed excesses, while also reaching significantly deeper contrast levels. Indeed, PFN measurements already strongly constrain the maximum level of emission due to various potential source geometries around Vega ${ }^{12}$. At Keck, such measurements will be possible on mag. 7 stars, with $10^{-4}$ detection limits for stars brighter than $\mathrm{m}_{\mathrm{K}} \approx 4$, a significant step forward. A rotating baseline nuller will thus be able to survey main sequence stars for hot debris disks, brown dwarfs and stellar secondaries at emission levels typically ten times fainter than is currently possible at $2 \mu \mathrm{m}$. The exoplanet (Hot Jupiter) case will moreover be aided by longer wavelengths (L band). Finally, with the nearly $30 \mathrm{~m}$ baselines possible on the TMT, a rotating nuller on that telescope will be able to provide clear views of the interiors of nearby solar systems down to within a few mas of the stellar position (thus including the dust sublimation region in a few cases), thus potentially uncovering the nature of any near-IR excesses around nearby main sequence stars, whether from dust or Hot Jupiters.

Finally, this work also has significant hardware implications, including a number of potential simplifications to future space-based nulling interferometers. In particular, the fiber nuller approach simplifies the beam combiner hardware, while the statistical data analysis approach leads to a relaxation of the fringe stability requirement, thus allowing less demanding control systems. On the other hand, the use of nulling techniques behind an ExAO system in the groundbased case allows the use of significantly shorter baselines than in long-baseline interferometry, thus enabling nulling measurements using large single apertures instead.

We thank the staffs of the Palomar and Keck Observatories for assistance with instrumental setups and with the observations. The research described herein was carried out in large part at the Jet Propulsion Laboratory, California Institute of Technology, under contract with NASA.

\section{REFERENCES}

[1] Bracewell, R.N. 1978, Nature, 274, 780

[2] Serabyn, E. and Mennesson, B. 2006, in Proc. IAU Coll. 200, "Direct Imaging of Exoplanets: Science and Techniques," eds. C. Aime and F. Vakili, p. 379

[3] Serabyn, E. and Mennesson, B. 2006, in 2006 IEEE Aerospace Conference Proceedings, paper 6.09.02, DOI 10.1109/AERO.2006.1655938

[4] Mennesson, B. et al. 2006, Proc. SPIE Vol. 6268, 96

[5] Martin, S.R. et al. 2008, Proc. SPIE Vol. 7103, 70131Y-1

[6] Guyon, O. et al. 2006, Ap. J. Suppl. 167, 81

[7] Angel, J.R.P. and Woolf, N.J. 1997, Ap. J. 475, 373

[8] Serabyn, E. 2000, in Proc. SPIE Vol. 4006, 328

[9] Haguenauer, P. and Serabyn, E. 2006, Appl. Opt. 45, 2749

[10] Hanot, C. et al. 2010, in prep.

[11] Mennesson, B. et al., this conference.

[12] Mennesson, B. et al., 2010, in prep.

[13] Absil, O. et al. 2006, A\&A 452, 237

[14] Absil, O. et al. 2008, A\&A 487, 1041

[15] Absil, O. et al. 2009, Ap. J., 704, 150

[16] Akeson, R. et al. 2009, Ap. J. 691, 1896

[17] Di Folco E. et al. 2007, A\&A 475, 243 